\title{
Survival analysis of adjuvant chemotherapy with S-1 plus cisplatin for stage III gastric cancer
}

\author{
Daisuke Takahari · Tetsuya Hamaguchi $\cdot$ Kenichi Yoshimura $\cdot$ Hitoshi Katai $\cdot$ \\ Seiji Ito · Nozomu Fuse · Masaru Konishi · Hirofumi Yasui • Masanori Terashima • \\ Masahiro Goto $\cdot$ Nobuhiko Tanigawa $\cdot$ Kuniaki Shirao $\cdot$ Takeshi Sano $\cdot$ Mitsuru Sasako
}

Received: 4 December 2012/Accepted: 21 April 2013/Published online: 30 May 2013

(C) The International Gastric Cancer Association and The Japanese Gastric Cancer Association 2013

\begin{abstract}
Background We previously reported that S-1 plus cisplatin was feasible as adjuvant chemotherapy for stage III gastric cancer after D2 gastrectomy. Herein we evaluate the recurrence-free survival and overall survival rates as secondary endpoints based on updated follow-up data.

Methods Patients with stage III gastric cancer who underwent D2 gastrectomy were enrolled. Treatment consisted of 3 cycles of $\mathrm{S}-1\left(40 \mathrm{mg} / \mathrm{m}^{2} \mathrm{PO}\right)$ twice daily on days $1-21$ and cisplatin $\left(60 \mathrm{mg} / \mathrm{m}^{2} \mathrm{IV}\right)$ on day 8 , and $\mathrm{S}-1$
\end{abstract}

D. Takahari $(\bowtie)$

Department of Clinical Oncology, Aichi Cancer Center Hospital,

1-1 Kanokoden, Chikusa-ku, Nagoya 464-8681, Aichi, Japan

e-mail: dtakahari@aichi-cc.jp

T. Hamaguchi

Gastrointestinal Oncology Division, National Cancer Center

Hospital, Tokyo, Japan

K. Yoshimura

Translational Research Center, Graduate School of Medicine,

Kyoto University Hospital, Kyoto, Japan

H. Katai

Gastric Surgery Division, National Cancer Center Hospital,

Kashiwa, Japan

S. Ito

Department of Gastroenterological Surgery,

Aichi Cancer Center Hospital, 1-1 Kanokoden,

Chikusa-ku, Nagoya 464-8681, Aichi, Japan

N. Fuse

Division of Gastrointestinal Oncology and Digestive Endoscopy,

National Cancer Center Hospital East, Kashiwa, Japan

M. Konishi

Division of Surgical Oncology,

National Cancer Center Hospital East, Kashiwa, Japan was given on days 1-28 every 6 weeks until 1 year after surgery.

Results From August 2007 to September 2009, 63 patients were accrued. Overall, 34 and 25 patients had stage IIIA and IIIB disease, respectively. After a median follow-up of 3.9 years, 16 patients experienced recurrence and 11 patients died. The 3-year recurrence-free survival rate was $74.1 \%$ (95 \% CI: $60.8-83.5 \%$, IIIA $81.8 \%$, IIIB $64.0 \%$ ). The 3 -year overall survival rate was $84.5 \%$ (95\% CI: $72.3-91.6 \%$, IIIA $87.9 \%$, IIIB $80.0 \%$ ).

H. Yasui

Division of Gastrointestinal Oncology, Shizuoka Cancer Center,

Shizuoka, Japan

M. Terashima

Division of Gastric Surgery, Shizuoka Cancer Center,

Shizuoka, Japan

M. Goto

Cancer Chemotherapy Center, Osaka Medical College,

Takatsuki, Japan

N. Tanigawa

General and Gastroenterological Surgery, Osaka Medical

College, Takatsuki, Japan

K. Shirao

Department of Medical Oncology, Faculty of Medicine,

Oita University, Yufu, Japan

T. Sano

Department of Surgery, Cancer Institute Hospital, Japanese

Foundation for Cancer Research, Tokyo, Japan

M. Sasako

Department of Surgery, Hyogo College of Medicine,

Nishinomiya, Japan 
Recurrence sites included the peritoneum $(n=8)$, hematogenous sites $(n=6)$, and lymph nodes $(n=4)$.

Conclusion The present results indicate that adjuvant therapy with S-1 plus 3 cycles of cisplatin may provide a survival benefit to patients with stage III gastric cancer.

Keywords Adjuvant chemotherapy - Gastric cancer . S-1 - Cisplatin

\section{Introduction}

In 2007, the Adjuvant Chemotherapy Trial of S-1 for Gastric Cancer (ACTS-GC) demonstrated the efficacy of S-1 for stage II-III Gastric Cancer (GC) patients who underwent curative resection with $\mathrm{D} 2$ gastrectomy [1, 2]. The addition of S-1 improved the overall survival (OS) rate, with a low incidence of adverse events and good compliance. According to this result, in Japan, the currently recommended adjuvant treatment after D2 gastrectomy is S-1 for 1 year. However, the 5-year OS rates in stage III patients receiving $S-1$ have been less satisfactory: 67.1 and $50.2 \%$ for stage IIIA and IIIB, respectively. Therefore, identification of more effective treatments for stage III GC is urgently needed. So firstly we evaluated the feasibility of S-1 plus cisplatin, that is now considered to be one of the standard regimens for metastatic or recurrent GC [3] as adjuvant chemotherapy for Stage III GC after D2 gastrectomy.

As results, treatment completion rates after 3 cycles of S-1 plus cisplatin were $72 \%(42 / 58 ; 95 \%$ CI: $60-84 \%$; $57 \%$ [12/21] before and $81 \%$ [30/37] after the protocol amendment). Grade 3/4 toxicities included neutropenia (40\%), anorexia (28\%), and febrile neutropenia (4\%) before the protocol amendment, and neutropenia (37\%), anorexia (8\%), and febrile neutropenia (3\%) after the amendment implementation. Therefore, we concluded that the amended S-1 plus cisplatin regimen is feasible as adjuvant chemotherapy [4].

In this report, we evaluate the recurrence-free survival (RFS) and OS as secondary endpoints based on updated follow-up data.

\section{Methods}

Patients eligible for this trial had either stage IIIA (T2,N2; T3,N1; T4,N0) or stage IIIB (T3,N2; T4,N1) [5] gastric adenocarcinoma and had undergone D2 gastrectomy with R0 surgical resection. Additional details were described as previously [4]. The protocol was approved by the institutional review board at each participating center. Treatment according to the original protocol was initiated 4-8 weeks after surgery with 3 cycles of S-1 plus cisplatin (SP) followed by S-1 for up to 1 year. In the SP step, each cycle consisted of $40 \mathrm{mg} / \mathrm{m}^{2} \mathrm{~S}-1$ taken orally twice-daily for 21 days plus a 2-hour infusion of $60 \mathrm{mg} / \mathrm{m}^{2}$ cisplatin on day 8 . Each cycle was administered at 5-week intervals. In the S-1 step, $40 \mathrm{mg} / \mathrm{m}^{2} \mathrm{~S}-1$ was taken for 28 days at 6-week intervals. During enrollment, some toxicity was reported during the first cycle of SP, particularly neutropenia and anorexia. To minimize patient's risk, we elected to amend the protocol. Treatment according to the amended protocol was initiated 4-6 weeks after surgery and consisted of the following: the first cycle of chemotherapy consisted of S-1 monotherapy, and cisplatin was added to cycles 2, 3, and 4. After that, S-1 was administered for up to 1 year. Tumor assessments with ultrasonography, computed tomography, and GI endoscopy and radiography were performed every 6 months for first 2 years after surgery, and annually thereafter (maximum follow-up 5 years). RFS was defined as the time from enrollment to the recurrence or death, whichever occurred first. OS was defined as the time from enrollment to death from any cause.

\section{Results}

From August 2007 to July 2009, 63 patients ( 25 patients in the original protocol, 38 patients in the amended protocol) were accrued from five Japanese institutions. Overall, 34 patients (54\%) had stage IIIA disease and $25(40 \%)$ had stage IIIB disease. The patient clinical characteristics have been reported previously [4]. After enrollment, 5 patients were deemed ineligible due to confirmed stage II disease $(n=2)$, stage Ib disease $(n=1)$, stage IV disease $(n=1)$, and cancer other than GC $(n=1)$.

OS and RFS were analyzed in 58 eligible patients. At the time of data cut-off on July 31, 2012, 11 patients had died, 5 patients were alive with recurrence, and the remaining 42 patients were alive without recurrence. The median follow-up period was 46 months. All patients could be followed-up for at least 3 years from the date of surgery. Kaplan-Meier estimates are shown that the 3-year OS rate was $84.5 \%$ (95\% CI: 72.3-91.6\%) (Fig. 1a), and the 3-year RFS rate was $74.1 \%$ (95\% CI: 60.8-83.5\%) (Fig. 1b). According to disease stage, the 3-year OS rate of patients with stage IIIA disease was $87.9 \%$ (95\% CI: 70.9-95.3\%) (Fig. 2a), and the 3-year RFS rate was $81.8 \%$ (95 \% CI: 63.9-91.4\%) (Fig. 2b). The 3-year OS rate of patients with stage IIIB disease was $80.0 \%$ (95\% CI: 58.4-91.1\%) (Fig. 2a). The 3-year RFS rate was $64.0 \%$ (95\% CI: 42.2-79.4\%) (Fig. 2b).

In addition, there was no significant difference in survival between the original protocol and the amended 
a

(\%)
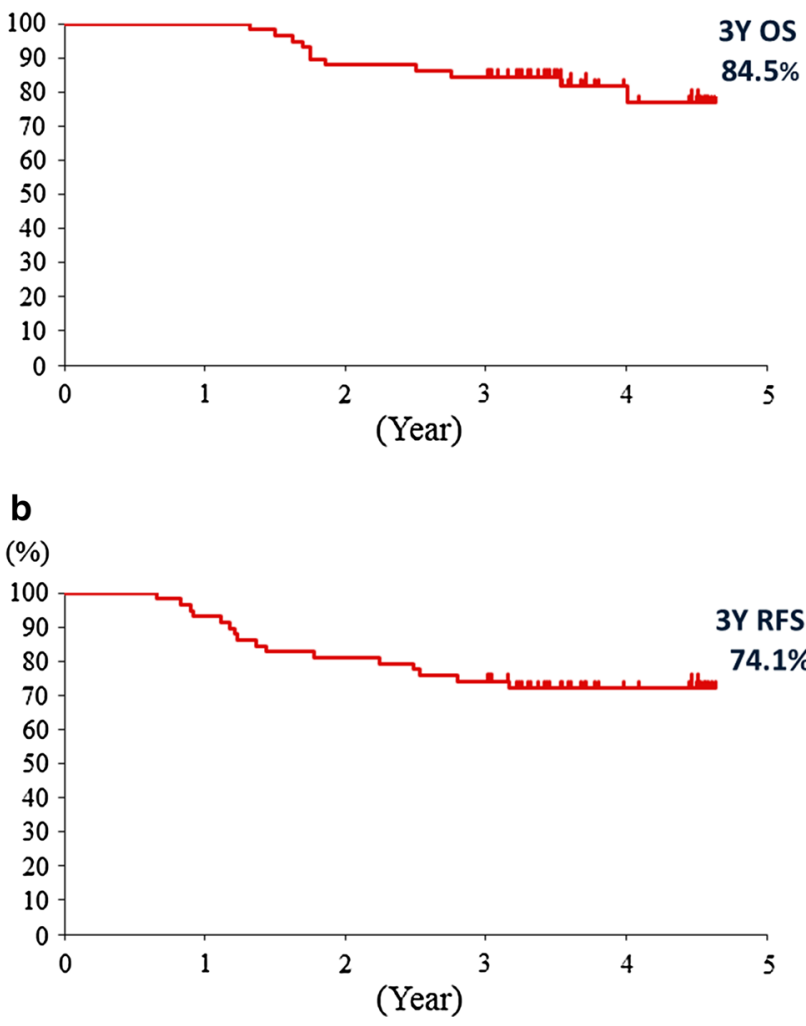

Fig. 1 Kaplan-Meier estimates of a overall survival and b relapsefree survival for all eligible patients

protocol. The 3-year OS rate of patients with stage IIIA disease in the original protocol $(n=16)$ and the amended ( $n=17$ ) was 87.5 and $88.2 \%$, respectively, and the 3 -year RFS rate was 75.0 and $82.4 \%$, respectively. The 3-year OS rate of patients with stage IIIB disease was $80.0 \%$ in the original protocol $(n=5)$ and the amended protocol $(n=20)$, and the 3-year RFS rate was 60.0 and $65.0 \%$, respectively.

The most common sites of relapse were the peritoneum ( $n=8)$, hematogenous sites $(n=6)$, and lymph nodes $(n=4)$. Two patients experienced relapses simultaneously in the liver and the lymph nodes. No local relapse was observed. After relapse, the median survival time was estimated to be 351 days. Subsequent therapies were taxanes $(n=7)$, SP $(n=4)$, S-1 $(n=3)$, and CPT-11 $(n=1)$, and 1 case underwent surgery (oophorectomy) followed by paclitaxcel.

\section{Discussion}

In this study, postoperative S-1 plus 3 cycles of cisplatin demonstrated promising efficacy with respect to 3-year RFS and OS for stage III GC.

\section{a}

(\%)

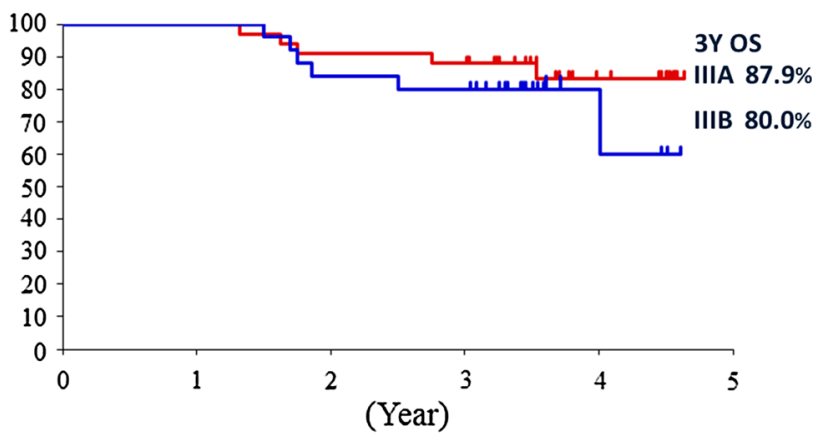

b

(\%)

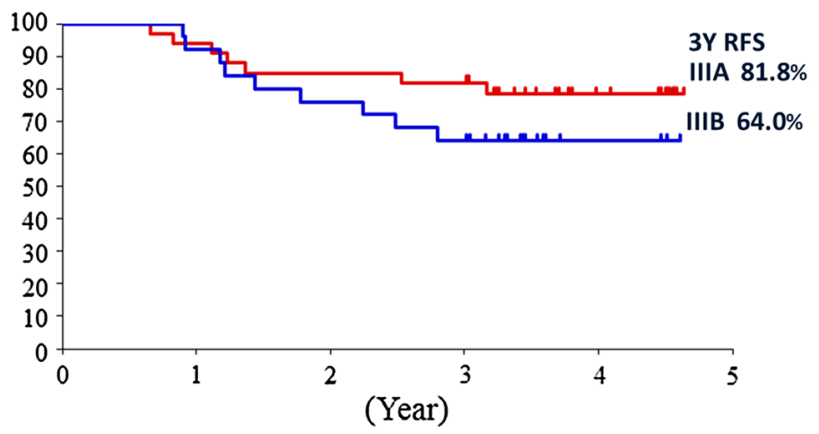

Fig. 2 Kaplan-Meier estimates of a overall survival and $\mathbf{b}$ relapsefree survival for patients with stage IIIA and IIIB gastric cancer

Recently, the results of the CLASSIC trial indicated that adjuvant capecitabine and oxaliplatin improved 3-year disease-free survival (DFS) compared with surgery alone in GC patients [6]. The subgroup analysis suggested that combined capecitabine and oxaliplatin were beneficial not only for stage II patients but also for stage IIIA and stage IIIB patients (the hazard rates compared to surgery alone were 0.57 and 0.57 , respectively). This result suggests that combination therapy with fluoropyrimidine and a platinum agent may be more beneficial than fluoropyrimidine alone in patients with stage III disease after D2 gastrectomy.

Although small-sample comparisons should be made with caution, there was no significant difference in survival between the original protocol and the amended protocol. It is suggested that delay of cisplatin administration in our amended protocol didn't sacrifice the efficacy in terms of survival. Consequently, we believe that completion of 3 cycles of cisplatin is important, even though we changed the first cycle to S-1 monotherapy and delayed additional cisplatin until cycles 2, 3, and 4. Moreover, our amended protocol was beneficial in the reduction of grade 3/4 anorexia and nausea, even though we did not use NK-1 receptor antagonists, because they were not approved in Japan at that time. Now we could manage the 
cisplatin-induced emesis easier by using NK-1 receptor antagonists with this regimen.

In conclusion, adjuvant therapy with $\mathrm{S}-1$ plus 3 cycles of cisplatin may reduce recurrence and improve survival in patients with stage III GC who underwent D2 gastrectomy. This treatment should be considered for use as an experimental arm for comparison to $\mathrm{S}-1$ in future postoperative adjuvant phase III trials.

Acknowledgments We thank Mr. Yushi Nagai, Ms. Michiyo Tada and Ms. Junko Ikeda for help in collecting and organizing the database. We received no financial support.

Conflict of interest The authors have declared no conflicts of interest.

\section{References}

1. Sakuramoto S, Sasako M, Yamaguchi T, Kinoshita T, Fujii M, Nashimoto A, et al. Adjuvant chemotherapy for gastric cancer with S-1, an oral fluoropyrimidine. N Engl J Med. 2007;357:1810-20.
2. Sasako M, Sakuramoto S, Katai H, Kinoshita T, Furukawa H, Yamaguchi T, et al. Five-year outcomes of a randomized phase III trial comparing adjuvant chemotherapy with S-1 versus surgery alone in stage II or III gastric cancer. J Clin Oncol. 2011;29: 4387-93.

3. Koizumi W, Narahara H, Hara T, Takagane A, Akiya T, Takagi M, et al. S-1 plus cisplatin versus S-1 alone for first-line treatment of advanced gastric cancer (SPIRITS trial): a phase III trial. Lancet Oncol. 2008;9:215-21.

4. Takahari D, Hamaguchi T, Yoshimura K, Katai H, Ito S, Fuse N, et al. Feasibility study of adjuvant chemotherapy with S-1 plus cisplatin for gastric cancer. Cancer Chemother Pharmacol. 2011;67: 1423-8.

5. Japanese Gastric Cancer Association. Japanese Classification of Gastric Carcinoma-2nd English Edition. Gastric Cancer. 1998;1: $10-24$.

6. Bang YJ, Kim YW, Yang HK, Chung HC, Park YK, Lee KH, et al. Adjuvant capecitabine and oxaliplatin for gastric cancer after D2 gastrectomy (CLASSIC): a phase 3 open-label, randomised controlled trial. Lancet. 2012;379:315-21. 